comunes, planes de investigación didáctica sobre temas comunes, materiales didácticos de apoyo a la enseñanza, etc...

En estos Coloquios participaron más de 250 profesores de Química de enseñanza superior y de secundaria de 10 países, aunque la mayoría eran de procedencia portuguesa, francesa y española. Precisamente estas tres lenguas junto a la italiana fueron declaradas oficiales en estas Jornadas.

El programa incluyó conferencias plenarias, seminarios, comunicaciones, mesas redondas y presentación de paneles y vídeos. Como ejemplo de conferencia puede mencionarse la realizada por M. Gomel que titulo sugerentemente "(De)formación pedagógica de los profesores de enseñanza superior", y cuyo mensaje fundamental lo basó en la necesidad de fomentar la formación pedagógica específica en la enseñanza superior, en desterrar la idea simplista de que enseñar es fácil y en aquella otra que argumenta que al profesorado universitario sólo se le debe juzgar la competencia en la direccion de investigaciones científicas. Otros ejemplos a citar son las desarrolladas por el profesor S. Senent de la UNED de Madrid, centrada en aspectos metodologicos de esta modalidad de enseñanzas y por el profesor J. Casado de la Universidad de Salamanca sobre el papel esencial que una investigación didáctica de mejor calidad puede desempeñar en la enseñanza de la Química.
También se llevaron a cabo seminarios interesantes como al de resolución de problemas sencillos dirigido por las profesoras Pestana y Pereira, el de la profesora M. L. Veiga de la Escuela Superior de Educación de Coimbra que utilizó los conflictos entre experiencia sensorial y teorías científicas en la formación del profesorado, o el del profesor A. Cachapuz de la Universidad de Aveiro, centrado en el análisis de los discursos utilizados por los estudiantes en los exámenes de Química. Sobre el establecimiento de proyectos cooperativos se presentó un seminario dirigido, entre otros, por el profesor J. Casanova de la Universidad de Valladolid.

Como ejemplo de mesa redonda donde se trataba de resaltar la cooperación transnacional en materia de canales de información, se puede citar la moderada por la profesora Raquel Gonçalves, directora del Boletim de la Sociedade Portuguesa de Química y en la que intervinimos M. Guerin de la Universidad de Poitiers, por el SESDIC $y$ Mme. J. Dauchot-Weymeers de la Universidad Libre de Bruselas, por el RECLAF y el que suscribe por Enseñanza de las Ciencias. Presentamos aquí tres ejemplos concretos, pero bien distintos, de vectores de información, de interés didáctico para los enseñantes de la Química, resaltando la importancia de la comunicación en cualquier campo de la investigación científica.

Finalmente indicar que un resultado inmediato de estos Coloquios fue el establecimiento de un conjunto de proyectos cooperativos a desarrollar, tales como:

-Elaboración de un glosario de términos técnicos en las cuatro principales lenguas latinas y en inglés, en colaboración con lingüistas.

- Creación de un banco de fichas con experiencias de laboratorio para el nivel de los dos primeros años de universidad.

-Diseño de material de bajo costo para usar en la formación del profesorado de secundaria.

-Preparación de materiales didácticos (escritos, audiovisuales, etc...).

-Colaboración más estrecha entre las Sociedades de Química y su apertura a artículos de Educación escritos en otras lenguas latinas, con resúmenes largos en la de la revista.

-Interrelación de organismos y Centros de recursos dedicados a la enseñanza de estudiantes con necesidades especiales.

La evaluación de estos proyectos cooperativos puede llevarse a cabo en la próxima versión española de estos Coloquios que se han previsto realizar en Málaga en 1992.

\title{
CENTROS DE FORMACIÓN DEL PROFESORADO
}

\section{PERFECCIONAMIENTO DOCENTE A DISTANCIA}

Un curso para profesores en Química

\section{Bulwik, M., Lastres de García, L.}

Programa Prociencia (Conicet-Ministerio de Educación y Justicia), Argentina.

Una forma de ayudar al mejoramiento de la educación química en la escuela media es fomentar el perfeccionamiento de los docentes y ofrecerles nuevas ideas para llevar a la clase. Pero la amplitud de nuestro territorio hace que los docentes estén muy alejados unos de otros y de los centros educacionales. Por eso, una buena forma de llegar a ellos es a través del correo. En nuestro país ya se han realizado cursos de perfeccionamiento docente a distancia., con buenos resultados.

En 1985 se crea Prociencia con el fin de organizar cursos a distancia para profesores de enseñanza media. Estos cursos corresponden a diversas disciplinas: Matemática, Física, Química y Biología, además del curso de Pensamiento Científico que está dirigido a docentes de cualquier asignatura.

En el proyecto de Química, durante 1986, enviamos una encuesta a profesores de todo el país, a fin de saber si estaban interesados en un curso de Química Orgánica. El resultado fue positivo y nos dio información sobre los principales temas que los docentes solicitaban para tal curso. Intercambiando ideas con profesores e investigadores en Química Orgánica de la Universidad de Buenos 
Aires realizamos el diseño general del curso. Tuvimos en cuenta que además del destinatario directo del mismo, el docente-alumno, debía ser considerado un destinatario indirecto, ei aitumno de escuela secundaria. En consecuencia no apuntamos sólo al perfeccionamiento docente, en cuanto a un enriquecimiento en conocimientos, sino también, en 10 posible, al aspecto metodologico de la enseñanza de la Química. Por ello, escogimos algunos temas centrales y tratamos de desarrollarios en profundidad, de relacionarlos con la vida diaria y de dar diferentes ejemplos de cómo trabajarlos con los alumnos.

El curso fue dividido en tres estructuras: Fundamentos, Biomoléculas e Industrias, con cinco módulos cada una y desarroliadas en cinco volúmenes. Los módulos fueron escritos por profesionales directamente vinculados, por su actividad, con cada tema, procesados didácticamente para darles el estilo final y revisados por asesores científicos. De esta forma logramos un acercamiento de los investigadores a la problemática de la eđucación científica en la escuela secundaria.

El primer volumen del curso aparecio en el segundo semestre de 1987. Los docentes interesados pueden inscribirse y comenzar el curso en cualquier época del año. Reciben el material por correo a muy bajo costo, el que corresponde a una hora de cátedra de nivel terciario nacional. Siguen sus progresos enviándonos evaluaciones escritas y recibiendo nuestros comentarios sobre ellas. También pueden realizar consultas en forma telefónica, personal o por correo. Como parte del curso, pueden dar dos evaluaciones presenciales. La aprobación de las mismas les permite recibir el correspondiente certificado. También se les pide que evalúen el curso. En esta forma podremos saber si es necesario realizar modificaciones en el mismo, para mejorar su calidad. Por otra parte, el diseño del curso da la posibilidad de agregar, en el futuro, nuevos módulos sobre temas re lacionados con los ya desarrollados, si son solicitados por los docentes.

Como parte del Programa Prociencia, hemos realizado y seguiremos realizando encuentros presenciales no evaluativos, en diversos Iugares de nuestro pais. En dichos encuentros se desarrollan actividades experimentales y discusiones metodológicas, buscando, además, el intercambio de ideas entre los profesores.

En la actualidad (julio de 1989) son unos 400 docentes los que están realizando el curso. Las opiniones recibidas hasta el momento nos indican una acogida favorable y nos impulsan a seguir trabajando en esta forma de apoyo a la tarea docente.
AREA DE EDUCAÇAO APLICADA AS GEOCIENCIAS

Compiani, $M$.

Instituto de Geociencias - UNICAMP (Brasil).

\section{Presentación}

El Area de Educación Aplicada a las Geociencias (AEAG) del Instituto de Geociencias de la Universidad Estadual de Campinas (UNICAMP), Brasil, tiene por objetivos la producción y la difusión del conocimiento generado de forma interdisciplinar por las Geociencias y Ia Educación.

Las pesquisas del AEAG estuvieron desce el comienzo centradas en la problemática psico-pedagógica de la introducción del conocimiento geológico en los varios niveles de escolaridad, manteniendo presentes al mismo tiempo las cuestiones relacionadas con la epistemología, la historia, la comunicación y la producción de recursos didácticos en Geología.

Las consideraciones teóricas y las actividades prácticas en el campo de la metodología de la enseñanza llevaron a la forma actual de trabajo del AEAG, desarrollando programas y proyectos que, además de tener identidad propia, se caracterizan por el pionerismo, la complementariedad y la interdisciplinaridad en cuatro líneas de pesquisa: Metodología de la Enseñanza en Geociencias, Teoría del Conocimiento Geológico, Historia de las Ciencias Geologicas y Comunicacion en Geociencias.

La línea de Metodología de la Enseñanza en Geociencias (MEG) trata de los currículos, programas y metodología de la enseñanza de Geología y Geociencias en los varios niveles de la escolaridad (desde el elemental hasta el posgrado), como también de la formación y perfeccionamiento de los profesores e investigadores.

La línea de Teoría del Conocimiento Geológico (TCG) trata de los fundamentos del conocimiento geológico y sus relaciones con otros campos del conocimiento; de la naturaleza de la información y representación geologica y de la metodología de producción del conocimiento geológico.

La línea de Historia de las Ciencias Geologicas (HCG) trata de las condiciones de produccion, incorporación y uso de los conceptos, teorías y modelos cientificos e institucionales, así como de los métodos y técnicas de investigación empleados en la enseñanza y en la pesquisa geologica en Brasit.
La línea de Comunicacion en Geociencias (CG) trata de las formas y procesos de introducción del conocimiento geológico y de sus bases materiales (en los dominios verbal y no-verbal) e institucionales.

La orientación primordial del AEAG es ejercer la enseñanza como pesquisa. Así, en consonancia con sus objetivos, la producción técrico-científica del grupo de profesores pertenecientes al AEAG ha empezado de la interacción entre acciones prácticas, experiencias didácticas e investigaciones teoricas. En ese sentido, se unen, sistemáticamente, las preocupaciones socio-históricas y epistemológicas con el conocimiento geológico y con una manera peculiar de realizar su introducción y el proceso de enseñanza/aprendizaje.

EI AEAG fue oficialmente creado en 1983 y cuenta hoy con nueve docentes en régimen de dedicación exclusiva, siendo 8 maestros (algunos en fase final de doctorado) y 1 licenciado (completando la maestrua).

El AEAG mantiene constante relación con el Departamento de Metodología de Ia Enseñanza (DEME) de la Facultad de Educación (FE) de la UNICAMP. En el plano internacional, además del intercambio con la Withelm-Pieck-Universität Rostock (RDA), con la University of Keele (England) y con la University of Concordia (Canadá), se han procurado contactos con profesores de las Universidades de València (España), Autonoma de Barcelona (España), Genova (Italia), Venezuela y Costa Rica.

\section{Programas y Proyectos}

\section{I. Actividades permanentes}

a) Curso de Especialización en Enseñanza de Geociencias para nivel universitario en colaboración con el DEME/ FE/UNICAMP.

b) Actividades de enseñanza a nivel universitario que es un excelente laboratorio para experiencias e investigaciones educacionales.

c) Actividades de divulgación informal del conocimiento geológico en el Museo Dinámico de Ciencias de Campinas.

\subsection{Actividades actuales}

\subsubsection{Proyectos}

a) "Cursos de actualización para profesores de ciencias del nivel elemental verdades y mitos".

b) "La institucionalización de las ciencias geologicas en Brasil - 1808-1946".

c) "La historia de los museos brasileños 
de ciencias naturales, deI siglo XIX a las primeras décadas del siglo XX".

d) "Geología y apropiación del espacio urbano: elaboracion del concepto de es. pacio geológico".

e) "EI universo de la Geología Introductoria en Brasil".

f) "Evaluación de los cursos de especialización en enseñanza de Geociencias".

g) "Semiotica de los mapas geologicos".

h) "EI constructivismo y la Geología en la enseñanza de la ciencia".

i) "Proyecto de Enserianza de Geociencias" (PEGE), para nivel elemental.

j) "Proyecto de Enseñanza de Geología Introductoria" (PEGI), para nivel superior.

\subsubsection{Laboratorio}

a) Laboratorio de Producción de Recursos Didácticos en Geociencias (en proceso de instalación).

\subsubsection{Cursos}

a) Cursos de extensión universitaria para profesores de Ciencias y Geografía en escuelas primarias y secundarias pertenecientes a la red pública de enseñanza del Estado de Sāo Paulo.

\subsection{Actividades realizadas}

a) I Jornada sobre la enseñanza del contenido geológico para los niveles elemental y secundarjo (julio de 1983).

b) 2 Cursos de Especialización en Enseñanza de Geociencias para nivel universitario (1984/85 y 1986).

c) Colaboración en el Curso de Especialización en Enseñanza de Ciencias para nivel elemental (1986).

d) Asesoría para la reformulación del currículo de Ciencias para la enseñanza a nivel elemental en el Estado de Sāo Paulo (1986/87).

e) I Coloquio Brasileño de Historia y Teoría del Conocimiento Geologico (julio de 1988).

f) Simposio sobre Especialización en Enseñanza de Geociencias para nivel universitario (julio de 1988).

El AEAG está dispuesto a colaborar y mantener intercambio con profesores, pesquisadores y/o gnupos que manifiesten interés en los campos de conocimiento afines. Toda correspondencia debe ser dirigida a:

Area de Educaçao Aplicada às Geociências

Instifuto de Geociências - UNICAMP

C.P. 6152 - 13081 Campinas, São Paulo, Brasil

Fone: 0192-391097 FAX (0055) 192 394717

Carlos Alberto Lobão da Silveira Cunha

Conrado Paschoale

Ivan Amorosino do Amaral Maria Margaret Lopes Mariley Flória Simōes Gouveia Mauricio Compiani

Oscar Braz Mendonza Negräo

Pedro Wagner Gonçalves

Sitvia F. de Mendonca Fişueirôa

\section{NOTICIAS}

ASOCIACION CANARIA PARA LA ENSENANZA DE LAS CIENCIAS "VIERA Y CLAVIJO"

VI Congreso. Las Palmas, del 25 al 27 de septiembre de 1990. I.B. Pérez Galdós. Patrocinado por la Consejería de Educación.

\section{Objetivos}

Los congresos de la A.C.E.C. se proponen contribuir a la formación continua del profesorado de ciencias mediante su actualización científica y pedagógica, el intercambio de experiencias didácticas, la discusion de las dificultades de aplicación de éstas en el aula y el estímulo de la creación de gripos de trabajo.

Consideramos conveniente centrar nuestro VI Congreso en propuestas concretas de trabajo en el aula, fundamentadas en la investigación didactica y dirigidas a cualquier nivel de enseñanza:
Dirigirse a:

c/ Doctor Zamenhof, 7 bajo derecha. Tel. 2539 52. 38204 La Laguna (Tenerife).

PRIMERA EXPOSICION INTERNACIONAL. CUIDEMOS NUESTRO MUNDO (CNM) (I - EXPO - INT CNM - 1990)

Abril-Setiembre de 1990. Auspicia UNES CO ROSILAC.

El Proyecto Cuidemos Nuestro Mundo (CNM) auspiciado por UNESCO-ROST-
LAC, tiene el agrado de dirigirse a Ud., a fin de tener el honor de invitar muy especialmente a vuestra institución para participar de la Primera Exposición Internaciona Cuidemos Nuestro Mundo (I-EXPOINT-CNM-1990), a realizarse en el período Abril-Setiembre de 1990, según las especificaciones del Boletín Informativo adjunto.

La importancia a nivel mundial de la temática general de la I-EXPO-INT-CNM. Contaminación y Mejoramiento de la Calidad de Vida, asuntos de primordial preocupación de dodos los gobiernos, hace que este acontecimiento sea un escenario validido para convocar a todos los docentes e investigadores y estudiantes avanzados (próximos a recibir títulos) de vuestra institucion que deseen participar, haciendo con sus trabajos, propuestas y proyectos un excelente aporte para sus respectivas comunidades, inherentes a temas relacionados con la supervivencia del hombre y demás seres vivientes del planeta. 